\title{
In vivo functional imaging with SPECT and PET
}

\author{
By H. Herzog* \\ Institute of Medicine, Forschungszentrum Jülich GmbH, D-52425 Jülich, Germany
}

(Received December 10, 1999; accepted in revised form August 14, 2000)

\author{
Single photon emission computed tomography / \\ Positron emission tomography / Functional imaging / \\ Radiopharmaceuticals / Gamma camera
}

\begin{abstract}
Summary. Nuclear medicine methods permit the visualisation of a variety of metabolic and physiological processes all over the body. Although planar scintigraphy has been found useful for many questions, detailed spatial information about the diseased organ can only be obtained with tomographic methods. Dependent on the radionuclide involved, two different tomographic procedures are available: single photon emission computed tomography (SPECT) and positron emission tomography (PET). The first part of this paper describes shortly the historical development of these methods as well as their technical and methodological basics. To elucidate the large variety of possible applications, an overview of SPECT and PET procedures utilised in research as well as in clinical practice are presented. Furthermore, both methods are compared and their individual advantages are pointed out.
\end{abstract}

\section{Introduction}

During the last three decades new powerful imaging procedures have been developed in medicine to improve the diagnosis of diseases. The methods differ in their underlying physical basics, the achievable image and time resolution and in the kind of information they produce. Whereas the strengths of computed tomography (CT), magnetic resonance tomography (MRT) and ultrasound (US) lie in the display of structural information, the attractive characteristics of nuclear medicine methods are their ability to visualise a variety of metabolic and physiological processes all over the body. For this purpose, substrates which take part in the metabolic and physiological processes are labelled with radioactive nuclides. The measurement of the radiation emitted by these nuclides produces different kinds of information needed for the diagnosis. In order to observe the temporal as well as the spatial dimension of the information tomographic methods have to be applied. Dependent on the radionuclide involved, two different tomographic procedures are available in nuclear medicine: single photon emission computed tomography (SPECT) and positron emission tomography (PET). The purpose of this review is to describe shortly the historical development of these methods as well as their technical and methodological basics. To elucidate the large

\footnotetext{
*E-mail: h.herzog@fz-juelich.de
}

variety of possible applications, examples of SPECT and PET procedures utilised in research as well as in clinical practice are presented.

\section{Historical development of SPECT and PET}

The original suggestions towards SPECT and PET were already made in the early 1960s by Kuhl and Edwards [1] and by Rankowitz and co-workers [2]. A successful introduction of these approaches was, however, hindered by insufficient reconstruction algorithms available at that time. Satisfying algorithms became available with the invention and early development of CT by Cormack [3] and Hounsfield [4]. The first commercial SPECT systems were developed in the late 1970s, when conventional gamma cameras used for planar scintigraphy were put on a special stand allowing the rotation of the gamma camera around a supine patient. Especially for cerebral applications, ring detector systems became available. To improve the sensitivity of SPECT present commercial systems use two, three or even four gamma cameras. Besides the improved sensitivity the systems have become more and more mechanically stable so that they are utilised in clinical routine with measurement times down to 10 minutes.

Parallel to the development of SPECT, PET devices were introduced - first mainly in research environments where necessary cyclotrons for the production of the short-lived positron emitters were often available. Most of those systems used ring or ring-like assemblies of sodium iodide (NaI) detectors surrounding the patient. There has been a steady improvement in PET systems with respect to sensitivity and image resolution by using BGO crystals instead of NaI crystals, by introducing block detector schemes and 3-dimensional acquisition techniques.

\section{Physical and technical basics of SPECT and PET}

\section{The detector system}

The primary element of each nuclear medicine detector system is the scintillation detector. Fig. 1a shows such a detector which consists of a scintillation crystal and a photomultiplier at least. The scintillation crystal is sensitive to the energy of $\gamma$-radiation that is emitted by radionuclides used in nuclear medicine, no matter whether they are single photon or positron emitters. The $\gamma$-radiation excites the scintillator material so that it emits light photons, a part of which hits the 


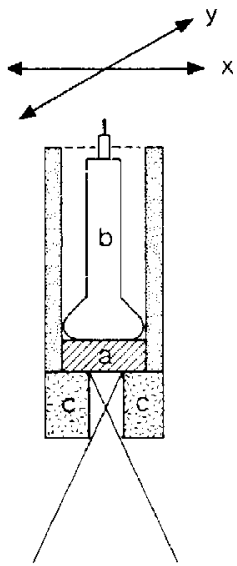

a)

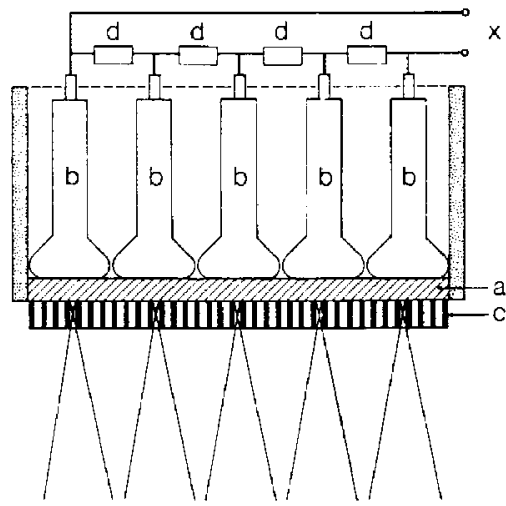

b)
Fig. 1. A scintillation detector (left) consists of a scintillation crystal (a), a photomultiplier (b), and a collimator (c) which restricts the direction of the detected radiation. The gamma camera (right) consists of an array of photomultipliers (b) mounted on a single NaI-scintillation crystal (a) and a collimator (c). A network of resistors (d) is used to weight the pulses of the different photomultipliers in order to localise (x) the viewed radiation.

photocathode of the photomultiplier where they cause a photoeffect producing electrons. These electrons are amplified within the photomultiplier tube resulting in a voltage pulse which is proportional to the number of light photons produced within the scintillation crystal and in this way proportional to the energy of the incoming $\gamma$-radiation. Fig. 2 shows the energy spectrum of the single photon emitter ${ }^{99 m} \mathrm{Tc}$. The energy spectrum of this emitter with only one single $\gamma$-energy consists of two parts. The photopeak has its maximum at the $\gamma$-energy of the radionuclide. The Compton spectrum represents the energy distribution of those $\gamma$-photons that have lost part of their energy by Compton scattering within the ob-

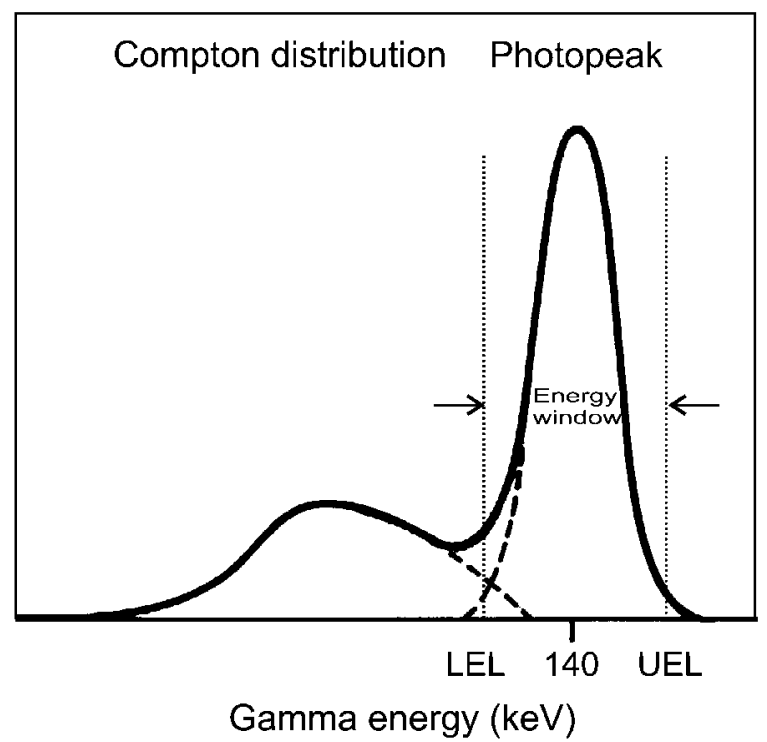

Fig. 2. Energy spectrum of the gamma emitter ${ }^{99 m}$ Tc consisting of photopeak and Compton spectrum. The energy window of the gamma camera with its lower energy level (LEL) and its upper energy level (UEL) is centred at the maximum of the photopeak. There is an overlap between the Compton distribution and the photopeak. ject to be investigated or in the scintillation crystal. In order to separate the true $\gamma$-radiation from that which has changed its direction by Compton scattering, discrimination electronics is used to distinguish between photopeak and Compton distribution. The discriminator circuit defines a lower and upper energy level so that the energy window within these levels includes the photopeak. The measurement of the radiation event by the scintillation detector must be completed by the information about the direction of the radiation, if an image of the activity distribution within the body is to be constructed. In the case of single photon emitters this information is obtained with the help of lead or tungsten collimators which allow only that radiation to reach the detector which comes from a direction specified by the collimator holes (Fig. 1). Data about uptake and outwash of activity into and out of this organ, i.e. counts as a function of time, can be obtained already with single scintillation detectors which are positioned over an organ of interest. In order to get twodimensional image information an assembly of single scintillation detectors has to be used such as is available in a gamma camera (Fig. 1b). A gamma camera consists of one large NaIcrystal on which an array of photomultipliers is mounted [5]. The light photons produced by a $\gamma$-ray within the NaI-crystal are seen by several neighbouring photomultipliers. In order to localise the entry of the $\gamma$-ray the signals produced by the photomultipliers are weighted by an appropriate resistor network. Furthermore, a collimator in front of the NaI-crystal excludes nearly all radiation not coming from a direction perpendicular to the gamma camera surface. There are many different kinds of collimators, e.g. to look in an oblique direction. They will, however, not be discussed here.

Whereas the existence of a mechanical collimator is essential for measuring single photon emitters by planar scintigraphy or SPECT, they are not necessary for PET which utilises a so-called electronic collimation. Fig. 3 shows the physical basis of PET. Here, a positron is the primary result of the radioactive decay. Depending on its energy, which is specific for the individual radionuclide, it travels a short distance between less than $1 \mathrm{~mm}$ and several $\mathrm{mm}$ after which it is nearly at rest so that it is able to interact with an electron of the surrounding material. As a result both particles annihilate and produce a pair of photons which are emitted in opposite directions and have an energy of $511 \mathrm{keV}$, each corresponding to the rest energy of one electron. In PET, scintillation detectors are mostly assembled as a ring. If the two photons of a photon pair hit two detectors of a ring within a very short time window (e.g. $12 \mathrm{~ns}$ ) it is assumed that these photons belong to the same annihilation process (coincidence measurement, Fig. 3). Therefore, the location of the radioactive atom is regarded to be on the connecting line between the two detectors (=electronic collimation). In this way information about the direction of the $\gamma$-radiation is obtained without any mechanical collimation. Until some years ago there was still a collimation between neighbouring detector rings so that the $\gamma$-radiation was guided within one plane, thus allowing for a twodimensional (2D) acquisition mode only. Modern PET tomographs offer a three-dimensional (3D) acquisition mode for which detectors of neighbouring rings are also combined by the coincidence electronics $[6,7]$. Such 3D systems exhibit a $3-5$ fold increase of sensitivity over a $2 \mathrm{D}$ system. 


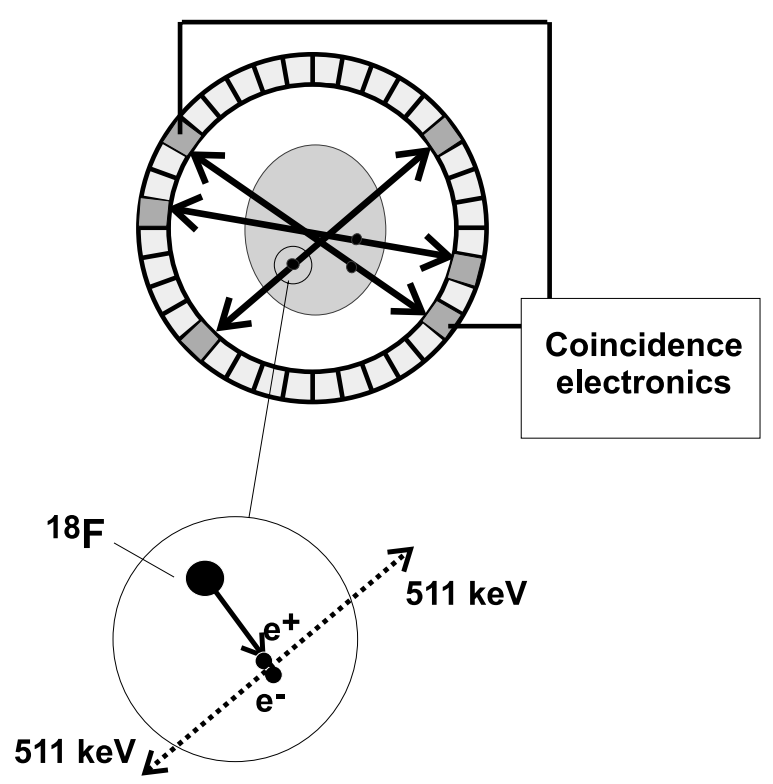

Fig. 3. Principle of coincidence measurement using a ring of scintillation detectors with opposing detectors connected by a coincidence electronics. The zoomed part scheme indicates the positron emission of the radionuclide ${ }^{18} \mathrm{~F}$ and the emission of two photons of $511 \mathrm{keV}$ after the positron meets an electron.

\section{Image reconstruction}

Whereas planar imaging, such as conventional radiology or planar scintigraphy, reduces the 3D distribution within the body to projected 2D information, tomographic procedures yield a fully $3 \mathrm{D}$ display. For this purpose projection data must be acquired from many views around the body which can be achieved by using a ring of detectors or by one or more gamma cameras rotating around the patient [8]. The basic relation which combines the projection data measured outside the body and the activity concentration within the body is given by:

$$
P(r, \alpha)=\int_{L(r, \alpha)} A(x, y) \mathrm{d} l(r, \alpha) .
$$

This kind of equation was first presented by the German mathematician Radon and is therefore called Radon transform [9]. It relates the 2D activity distribution $A(x, y)$ described in cartisian coordinates and the line integrals $P(r, \alpha)$ defined with polar coordinates. The line integral is the sum of all photons measured by a detector looking at the body with a specific angle $\alpha$. The exact view of the detector is defined by the polar coordinates $r$ and $\alpha$ (see also Fig. 7). Eq. (1) does not take into account the photons emitted by the radioactivity, but absorbed and scattered within the body. The amount of absorption and scattering is dependent on the density and shape off the body tissue and on the physical characteristics of the radionuclide. There are considerable differences in handling these effects with SPECT and PET. Provided these effects can be neglected or are satisfactorily corrected the task to conclude from the recorded projection data $P(r, \alpha)$ to the unknown activity concentration $A(x, y)$, i.e. the solution of (1) with respect to $A(x, y)$ is identical for SPECT and PET. The mostly used reconstruction method for obtaining $A(x, y)$ is the filtered backprojection [10]. According to the term backprojection the data recorded from different views $\alpha$ are continuously projected back into the $x, y$ plane. The superposition of all backprojected data, however, yields only a blurred image of the actual activity distribution. Therefore, this data must be sharpened by using an appropriate filter, i.e. a ramp filter which amplifies high rather than low frequency components (Fig. 4). The resulting image represents the desired activity concentration $A(x, y)$. In many applications filtered backprojection is the reconstruction method of choice, i.e. in all studies where sufficient photons are recorded by the radiation detectors and where the activity contrast between nearby structures of interest is not high. If these two prerequisites are not fulfilled, streak artifacts become visible. Such artifacts can be avoided with an alternative reconstruction method that calculates the activity image iteratively in an algebraic way. This method can be derived from a statistical and a numerical point of view, the latter of which is shortly described here. In this approach eq. (1) is rewritten as a product of matrices

$$
p_{n}=z_{m n} a_{m} .
$$

where $p_{n}$ is a matrix comprising all projection elements and $a_{m}$ represents the activity data at the image pixels $m$, i.e. the unknown activity distribution. The members of the matrix $z_{m n}$ describe in which way the coincidence events emitted from image pixel $m$ contribute to a single projection $p_{n}$. Commonly, this equation cannot be solved for the unknown matrix $a_{m}$. Instead, it is solved in an iterative way; for instance, by using an algorithm such as:

$$
a_{m}^{n+1}=a_{m}^{n} \sum z_{m n} \frac{p_{n}}{\sum z_{n m} a_{m}^{n}} .
$$

This equation represents the maximum likelihood (ML) expectation-maximisation (EM) method for iterative reconstruction [11]. The use of the iterative reconstruction is advised especially in the case of low counts and high contrast images. An earlier disadvantage of this method was the very long computation time - a problem which has been solved by fast computers and the introduction of acceleration parameters [12] as well as the ordered subset method [13]. The quantitative accuracy assured for the original ML-EM procedure may not be guaranteed for other solutions. Looking at the literature many different suggestions for iterative recon-

\section{Backprojection}

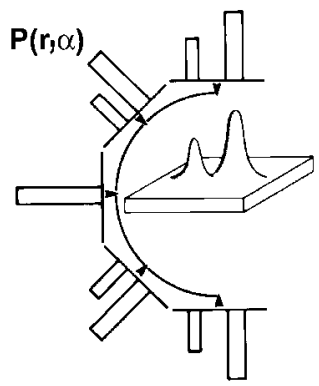

\section{Reconstructed object}

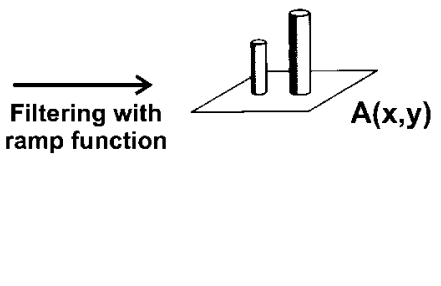

Fig. 4. Principle of filtered backprojection. The different projection data are backprojected into the imaging space and superimposed, resulting in a blurred object from which the desired reconstructed object is obtained after using ramp filtering. 
struction for both SPECT and PET applications have been published in recent years [14].

As already explained above, the ramp (high pass) filter is an integral part of the filtered backprojection and counterbalances the low pass characteristics of the mere backprojection. A severe disadvantage of high pass filtering is the amplification of statistical noise present in the measured counts. Therefore, the ramp filter is limited to low frequencies and/or multiplied with an appropriate window function to weaken the high pass characteristics of the ramp filter. Such window functions are the Hann, Shepp-Logan, Hamming, Parzen, or the Butterworth window. Although the reduction of the high frequency statistical noise is welcome, one has to consider that the image resolution becomes worse compared to the use of the original ramp filter. The decreased image resolution results in worse delineation of small details and a reduction of the image maxima. The decreased image resolution due to the use of a frequencylimiting window has a similar consequence as the use of a detector system with low intrinsic resolution. In both cases the partial volume effect increases. This effect causes an underestimation of the observed activity nearby low activities and an overestimation nearby high activity concentrations and is especially relevant for small structures. To reduce such errors in combination with a satisfying suppression of noise, the window functions and their upper frequency limit have to be selected carefully.

Although iterative reconstruction does not involve a filter problem as just discussed, some other characteristics must be taken into account. Generally, iterative reconstruction allows an image resolution near to the optimum scanner resolution. In practice, however, the final image resolution is dependent on the individual reconstruction algorithm and on its specific implementation. Therefore, the resolution performance of the individual program should be checked. A general rule is that the resolution becomes better with the number of iterations. Some iterative reconstruction procedures such as the ML-EM method exhibit, however, high frequency noise at many iterations, although the convergence of the method is guaranteed. Furthermore, the increase of noise is dependent on the specific program implementation. To avoid such noise, modifications of the reconstruction algorithms and/or a post processing filtering have been proposed. In both cases the image resolution becomes worse so that the partial volume effect is augmented and the quantitative accuracy of PET may be reduced [15].

In order to reconstruct data acquired with 3D mode, appropriate algorithms of 3D-FBP have been developed $[16,17]$. Although eq. (1) cannot exactly be solved for the case of 3D, several approaches for the 3D-FBP are successfully utilised with commercial PET systems. In the meantime their quantitative accuracy has been proven to be satisfactory. Furthermore, it is possible to rebin the 3D data in $2 \mathrm{D}$ sinograms so that they can be reconstructed as is known from the $2 \mathrm{D}$ acquisition mode. In the case of single slice rebinning the oblique projections are assembled into a $2 \mathrm{D}$ sinogram belonging to the plane in which they cross the central axis. The method of multislice rebinning distributes the oblique projections into the sinograms of those planes which are crossed by the oblique projection [18]. The Fourier rebinning method (FORE) applies a weighted distribution of the 2D sinograms so that the relationship between the direct and the oblique plains are considered in a theoretically exact way [19]. The 2D sinograms resulting from the different rebinning methods can be reconstructed by the conventional 2D filter backprojection or by an iterative reconstruction method.

\section{Correction procedures}

The information available in reconstructed SPECT or PET images should be linear to the underlying activity, i.e. a twofold activity concentration in a certain tissue must be represented by a twofold pixel value in the reconstructed image. On the other hand, the radiation emitted by the activity is distorted in several ways before it reaches the scintillation detectors and the acquisition electronics. On their way through the body tissue the $\gamma$-rays may be scattered and/or absorbed (Fig. 5). Furthermore, the dead time of the detectors might lead to erroneous results. In PET random coincidences have to be considered (Fig. 5b). In order to guarantee the linearity of radiation measurement these effects must be corrected [20].

\section{Attenuation correction}

As indicated by the following equations one of the major differences between SPECT and PET concerns the correction for those photons which have been absorbed within the body (Fig. 5).

The projection equation for SPECT reads

$$
\begin{aligned}
P(r, \alpha)=\int_{L(r, \alpha)} A(x, y) & \\
& \quad \times \exp \left(-\int_{L^{\prime}(r, \alpha)} \mu(x, y) \mathrm{d} l^{\prime}(r, \alpha)\right) \mathrm{d} l(r, \alpha),
\end{aligned}
$$
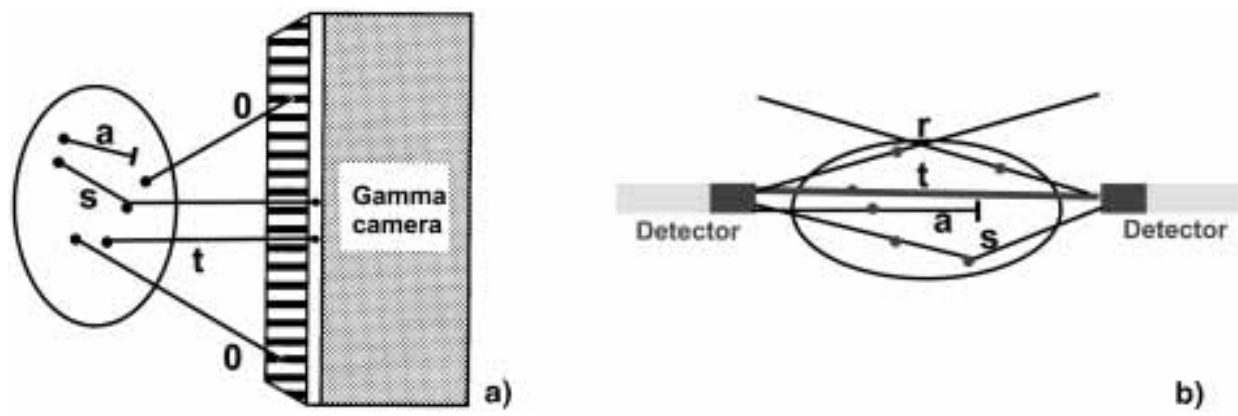

Fig. 5. Valid $(t=$ true) and erroneous radiation seen by a gamma camera and/or a coincidence detector pair: scattered radiation (s) and random coincidences (r). A part of the radiation is absorbed within the body (a). The gamma camera accepts only that information ( $\mathrm{t}$ ) which coincides with the direction of the collimator holes, whereas other photons are rejected (0). 
whereas the following equation describes the projections valid for PET

$$
\begin{array}{r}
P(r, \alpha)=\int_{L(r, \alpha)} A(x, y) \mathrm{d} l(r, \alpha) \\
\quad \times \exp \left(-\int_{L(r, \alpha)} \mu(x, y) \mathrm{d} l(r, \alpha)\right) \\
A F=\exp \left(-\int_{L(r, \alpha)} \mu(x, y) \mathrm{d} l(r, \alpha)\right) \\
P^{\text {corr }}(r, \alpha)=P(r, \alpha) / A F=\int_{L(r, \alpha)} A(x, y) \mathrm{d} l(r, \alpha) .
\end{array}
$$

The relationships of (5)-(7) are illustrated in Fig. 6. The decisive advantage of PET is that the integral over the activity distribution $A(x, y)$ and the exponential expression containing $\mu(x, y)$ are two factors of a product and can be determined in two steps, one after the other. In order to correct the photon attenuation, the attenuation factor $A F(6)$ is usually determined by a transmission measurement of, for example, $10 \mathrm{~min}$ mostly prior to the tracer injection. Because of this correction an equation (7) of the Radon form (1) is obtained and can be solved as described above. The attenuation correction is essential, if the PET measurement is to be quantitative. If this correction is not done, e.g. to shorten the acquisition time in whole-body studies, the PET results can only be judged qualitatively. Furthermore, without attenuation correction the reconstructed images show severe distortions. Activity concentrations being the same in the interior and at the border of the body, are imaged totally differently with an overestimation of the activity at the border. The PET image without attenuation correction is not linear. A new approach which aims at a very short transmission scan without loss of the signal to noise ratio is the use of single photon emitters instead of positron emitters for the transmission source. In this case more counts can be measured in a much shorter time. If the transmission measurement is done with positron emitters only, one photon is needed for the transmission information and the other photon is just used to define the line of response (see

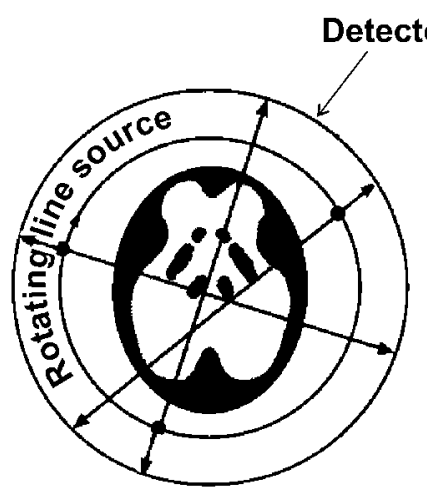

Transmission

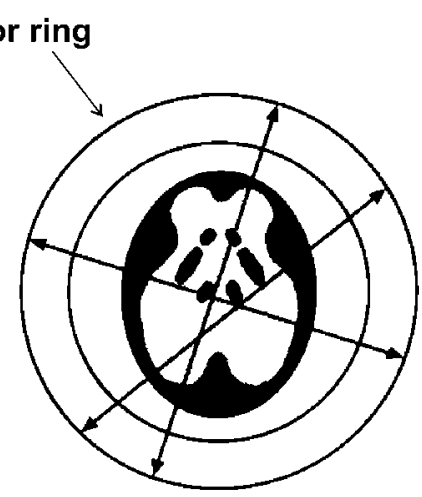

Emission
Fig. 6. In PET a transmission measurement yields attenuation factors to correct for the absorption on the same lines along which the emission scan records the coincidence events.
Fig. 6). If it is possible to define the line of response in another way, e.g. by tracking the path of the transmission source, positron emitters are no longer needed. Instead single photon emitters with a similar photon energy, but a much longer half-life can be utilised such as the single photon emitter ${ }^{137} \mathrm{Cs}$ with a photon energy of $662 \mathrm{keV}[21,22]$. Due to the higher photon energy, the measured attenuation data differ from that valid for the $511 \mathrm{keV}$, although the transmission image is practically identical. One way to correct this difference is to adjust the measured attenuation factors to the photon energy of $511 \mathrm{keV}$. Another way is to segment the reconstructed transmission image and to insert the standard attenuation coefficients for the different tissues. The last mentioned way results in noise-free attenuation factors AF. Using this approach the transmission time may be decreased to only 10 minutes for five bed positions. Furthermore, distortions between the different body parts are avoided.

In contrast to PET, a transmission scan done in SPECT does not directly enable an accurate attenuation correction, because the integration path $L^{\prime}(r, \alpha)$ reaching from the point of emission to the body surface remains unknown. Therefore, the projection equation for SPECT (4) cannot be solved analytically, e.g. by filtered backprojection. In some applications the attenuation term is just neglected assuming $\mu=0$, i.e. the attenuation coefficient of air. In spite of this simplification SPECT without attenuation correction proved to be very useful in many clinical applications, especially heart studies (see below). Looking at the brain or abdomen it may be justified to assume a constant $\mu$, namely that of water with a value of $0.154 \mathrm{~cm}^{-1}$. To allow a pre-reconstruction attenuation correction the projection data are acquired completely around the patient, then opposite views are geometrically averaged (Fig. 7). Because of this combination and by assuming a constant $\mu$ for the tomographic section to be reconstructed the following factor $A F$ for the co-called first order attenuation correction can be derived [23]:

$$
A F=(1-\exp (-\mu l)) / \mu l,
$$

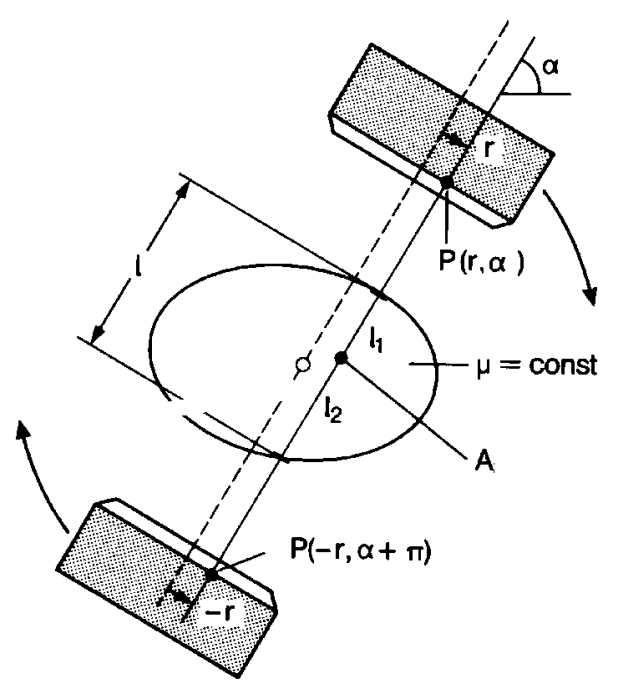

Fig. 7. Principle of measuring opposite projections to enable a first order attenuation correction of SPECT data. 
so that the projection equation for this approach reads:

$$
P^{\text {corr }}(r, \alpha)=P^{\text {geo }}(r, \alpha) / A F=\int_{L(r, \alpha)} A(x, y) \mathrm{d} l(r, \alpha) .
$$

Here similar to PET, the filtered backprojection sums projections just over one half-circle. Based on this approach a second order attenuation correction for SPECT data has been developed, introducing one or more (iterative) postprocessing steps to increase the accuracy of the correction [24].

\section{Scatter correction}

As shown in Fig. 5 a photon may change its direction within the body because of Compton scatter. Such photons cause a low-frequency background and decrease the image contrast. The primary means to eliminate them is energy discrimination. The Compton scatter results in a loss of energy so that these photons contribute to the Compton distribution shown in Fig. 2. The electronic energy discrimination defines an energy window around the photopeak where the lower threshold is to separate between scattered and nonscattered photons. As indicated in Fig. 2. there is always a small overlap. On the other hand the lower energy threshold must be set not too high in order not to lose too much unchanged photons - and sensitivity. The width of the energy window expressed as percentage of the mean photopeak energy is dependent on the light output of the scintillation crystal material. In this respect, NaI, which is used for SPECT, is more favourable than BGO. In spite of its wider energy window BGO is found in most PET-scanners, because its density is more adequate for the $511 \mathrm{keV}$ energy of the PET photons than that of $\mathrm{NaI}$.

Since it is not possible to exclude all scattered photons by energy discrimination additional procedures for correction have to be implemented. A lot of different methods have been suggested. The simplest procedure is to modify the first order attenuation correction by using a $\mu$ of $0.12 \mathrm{~cm}^{-1}$ instead of $0.154 \mathrm{~cm}^{-1}$. By weakening the attenuation correction the scatter correction is included in some way. A major approach is the dual-energy window technique. Here data are recorded in the photopeak window as well as in a window covering the Compton distribution. It is assumed that the scattered photons $P^{\text {Compton }}$ measured within the Compton window and within the photopeak have a fixed ratio $k$ so that a correction can be done according

$$
P^{\text {corr }}=P^{\mathrm{pp}}-k \cdot P^{\text {Compton }}
$$

where $P^{\mathrm{pp}}$ are all - nonscattered and scattered - events registered within the photo-peak [25]. A basic problem is that $k$ is very object-dependent. In addition to this simple method more sophisticated correction methods have been developed [26-28] so that the quantitative accuracy of SPECT has improved considerably for many applications.

Although being basically the same the scatter problem in PET differs in some aspects from that found in SPECT. Due to the higher photon energy the amount of scattered photons is less. On the other hand modern PET scanners offer the 3D-acquisition mode, where septa that prevent scatter are retracted so that $50 \%$ of the measured coincidences may be scattered. One correction method utilises the Klein-Nishina model of scatter and requires the additional transmission measurement to get knowledge about the scatter material [29]. An easier procedure has been suggested by Stearns [30]. For the 2D-measurement mode a point- source is measured in air and in water, so that a scatter function can be modelled and a convolution-subtraction-based correction model is applied [31]. In spite of the corrections there may be a residual scatter fraction of 5 to $10 \%$. A specific problem for 3D-PET is scattered radiation coming from outside the field of view. Here the ideal method would be the dual energy approach applied to each detector element which needs, however, a considerable increase in cost for electronics.

\section{Dead time correction}

All scintillation detectors exhibit a dead time effect if the measured count rate is high. The dead time is dependent on all (non-scattered and scattered) photons seen by the detectors. To correct the dead time a calibration measurement is performed which relates the counts originating from an activity source with the photons actually measured by the individual detector modules. In regard to PET the dead time is not just dependent on the rate of coincidences, but on all photons, which also include photons originating from activity outside the field of view.

\section{Correction for random coincidences}

This type of correction is specific for PET. A random coincidence occurs if two single photons belonging to two different positron emissions are registered by two opposing detectors within the coincidence time window and are falsely regarded as a coincidence event (Fig. 5b). The random coincidences cause a low frequent background. There are two procedures to correct the random coincidences. A hardware based method uses a delayed coincidence time window having the same length as the original coincidence window. All coincidences counted in the second interval are regarded as being random and directly subtracted from the measured (prompt) coincidences. The second method performs a calculated correction [32]. In this case the single count rates $S_{i}$ of the individual detectors are measured as well. Knowing these count rates and the duration $\tau$ of the coincidence time window the random coincidences $R$ are

$$
R=2 \tau S_{i} S_{j},
$$

which are subtracted from the measured coincidences as a prereconstruction step.

\section{SPECT applications}

Relevant clinical applications of SPECT imaging can be found for practically all organs of the body from brain over lung, heart, liver down to the legs. Looking at the literature most publications refer to cardiological and neurological questions. A specific characteristic of SPECT is that the acquisition time of SPECT with its detectors slowly rotating 
around the patient is longer than that of the PET ring detector systems. An essential prerequisite of SPECT is that the distribution of the radiopharmaceutical injected into the patient remains practically stationary during the detector rotation around the patient. Therefore, SPECT radiopharmaceuticals must be tailored so that after their uptake into an organ they show a slow outwash. Furthermore, the half-life of the radionuclide must be sufficiently long. For dynamic acquisitions a rotation time of $2 \mathrm{~min}$ may be appropriate to record a single time frame with a triple-head SPECT system, which yields high quality static image in $15 \mathrm{~min}$, for example.

The success of nuclear medicine in general, but also of SPECT is strongly coupled to an artificial radionuclide, namely ${ }^{99 m} \mathrm{Tc}$. It emits a monoenergetic single photon radiation of $140 \mathrm{keV}$ (see Fig. 2). Furthermore ${ }^{99 m}$ Tc has a convenient half-life of about 6 hours (not too short for purpose of storage and imaging and not too long for purpose of dosimetry), and is available with a generator system delivered weekly to the customer [33]. Therefore in contrast to PET, SPECT does not depend on a nearby cyclotron. Over the years the radiopharmaceutical industry has developed many kits which contain dry precursor compounds to be labelled with ${ }^{99 m} \mathrm{Tc}$. In addition, there are other photon emitters used, such as ${ }^{123} \mathrm{I}$ or ${ }^{201} \mathrm{Tl}$ with a half-life of $13.2 \mathrm{~h}$ and $3 \mathrm{~d}$, respectively.

A specific advantage of SPECT is the possibility of multitracer studies where different radiopharmaceuticals, each with another radionuclide (and a separate $\gamma$-energy), are injected at the same time or in combination. Practically all gamma-cameras allow multiple window studies with different energy windows so that a separation of the radionuclides is possible.

\section{Neurological SPECT}

The majority of SPECT imaging of the brain uses ${ }^{99 m} \mathrm{Tc}$ labelled HMPAO [34] or ECD [35] (sometimes still ${ }^{123}$ I-IMP [36] - especially in Japan) to trace the regional cerebral blood flow (rCBF). Mostly, decreases of rCBF are looked for, as they occur in a variety of brain disorders. Fig. 8 shows a typical example related to the diagnosis of Morbus Alzheimer. In Alzheimer's dementia a typical pattern of low perfusion in the region of the occipito-parietal cortex is found and can help in the differential diagnosis among other kinds of dementia. Also, in epilepsy, rCBFSPECT has shown its value [37]. If the rCBF-SPECT is done between epileptic seizures (inter-ictal), a localised decrease of tracer uptake is regarded as being concordant with the epileptic focus. If, however, the study is done during or immediately after an epileptic seizure (ictal and peri-ictal, respectively), the epileptic focus is delineated by increased blood flow. It has been shown that the subtraction of interictal images from ictal images yields an even better diagnostic sensitivity [38]. Immediately after brain infarction, rCBF-SPECT is able to show perfusion changes, in spite of a still normal CT. At a later stage areas, which show a decreased rCBF, indicate functional deficits caused by lacking input (afferences) from the infarcted region.

Although originally a domain of PET, more and more neurotransmitter studies are found in the SPECT literature.

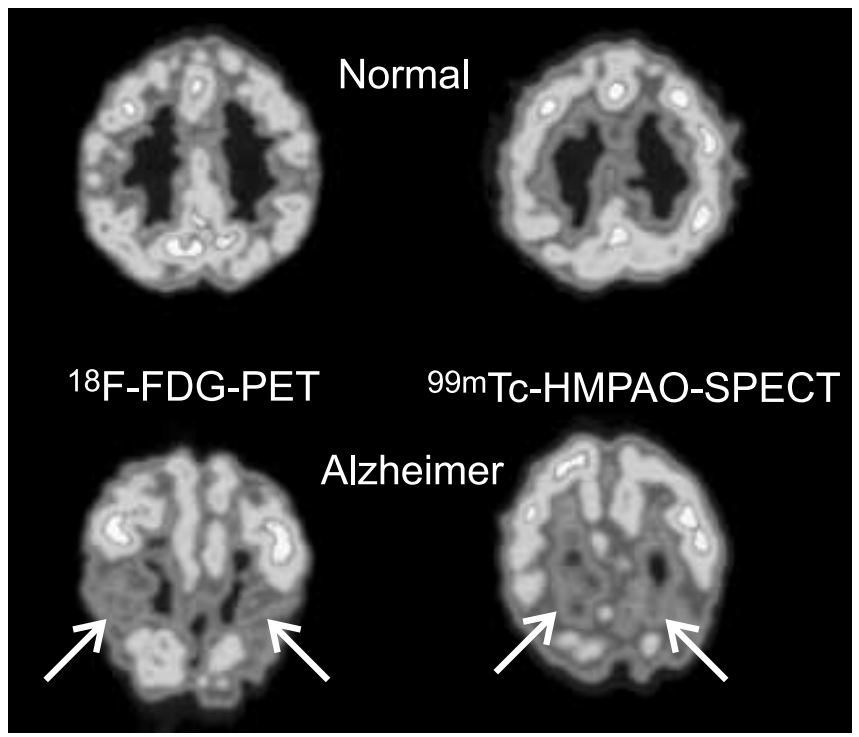

Fig. 8. In comparison to a normal subject there is less radioactivity in the occipito-parietal cortex of a patient with Alzheimer's disease (arrows). This finding can be visualised by SPECT using ${ }^{99 m} \mathrm{Tc}-\mathrm{HMPAO}$ (indicating decrease of blood flow) as well as ${ }^{18} \mathrm{~F}$-FDG-PET (indicating a corresponding decrease of glucose metabolism).

Most of these compounds are labelled with ${ }^{123} \mathrm{I}$. The size of this atom is appropriate to substitute a methyl group during the radiochemical labelling. In contrast to PET, where a labelling with ${ }^{11} \mathrm{C}$ does not change the biochemical behaviour of a pharmaceutical, an altered behaviour must be taken into account when using ${ }^{123} \mathrm{I}$. The long halflife of ${ }^{123}$ I may be an advantage over PET because the SPECT scan can be done many hours after the injection which might be favourable for some applications. A recent example is ${ }^{123} \mathrm{I}-\beta$-CIT, which is a tracer of the cerebral dopamine transport. It shows an optimum image quality one day after injection. ${ }^{123}$ I- $\beta$-CIT yields similar results as the PET tracer ${ }^{18} \mathrm{~F}$-L-Dopa by showing a decreased uptake in the striatum of patients with Morbus Parkinson [39]. Other ${ }^{123}$ I-labelled tracers are the dopamine ${ }_{2}$ receptors ligands ${ }^{123}$ I-IBZM [40] or ${ }^{123}$ I-IBF [41] and the central benzodiazepine receptor ligand ${ }^{123}$ I-iomazenil [42]. These tracers have been tested in various neurological and psychiatric disorders [43]. Many other ${ }^{123}$ I-labelled neurotransmitter tracers have been suggested during the last years, which cannot be detailed here.

${ }^{123}$ IMT, a radiolabelled amino acid, has proved to be very useful in the delineation of brain tumours [44] giving similar results as the PET tracer ${ }^{11} \mathrm{C}$-methionine (Fig. 9) [45]. Compared to FDG (see below) complementary information about the tumour extension may be obtained.

\section{Cardiological SPECT}

In daily clinical routine most SPECT studies deal with the heart. Here, radiotracers such as ${ }^{9{ }^{m}}$ Tc-MIBI [46], ${ }^{99 m} \mathrm{Tc}$-tetrofosmin [47], or still ${ }^{201} \mathrm{Tl}$ [48] are used to study myocardial perfusion, especially if there are signs of coronary artery disease, such as angina pectoris. The common protocol is to combine a rest study with a stress study so that badly perfused areas are delineated by decreased tracer uptake during the stress study with its increased energy de- 


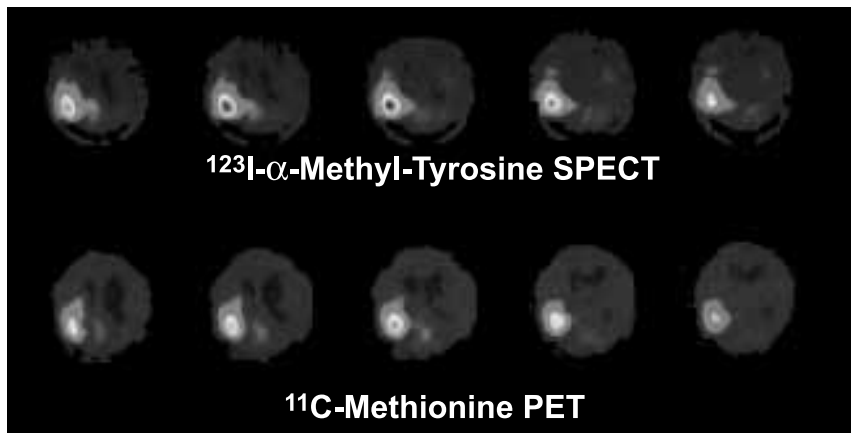

Fig. 9. Increased uptake of a radiolabelled amino acid in an astrocytoma grad II shown by SPECT using ${ }^{123} \mathrm{I}-\alpha$-methyl-tyrosine as well as by PET using the natural amino acid methionine which remains biochemically unchanged by the labelling with ${ }^{11} \mathrm{C}$.

mand. The stress is induced by exercising or dobutamine injection. Myocardial perfusion tracers may also be used to follow up the results after bypass surgery or percutaneous transluminal coronary angioplasty (PTCA). The advantages of ${ }^{99 m} \mathrm{Tc}$-labelled perfusion tracers compared to the former standard imaging agent ${ }^{201} \mathrm{Tl}$ are the better imaging quality and the availability of the generator product ${ }^{99 m} \mathrm{Tc}$.

${ }^{123}$ I-labelled fatty acids have been designed to examine myocardial energy metabolism which is based on the oxidation of fatty acids by about $60 \%$. ${ }^{123}$ I-HDA [49], ${ }^{123}$ I-BMIPP [50] and ${ }^{123}$ I-pPPA [51] and have been applied as a tool to investigate patients with cardiomyopathy [52].

Disorders of the autonomous myocardial innervation which are found, e.g., with dilative cardiomyopathy, heart transplantation, and diabetes have been examined with ${ }^{123}$ I-MIGB [53] (Fig. 10).

\section{Oncological SPECT}

Although the diagnosis and the follow up of malignant tumours is primarily the task of planar radiology, CT, MRT, or ultrasound, nuclear medicine can help in the case of un-

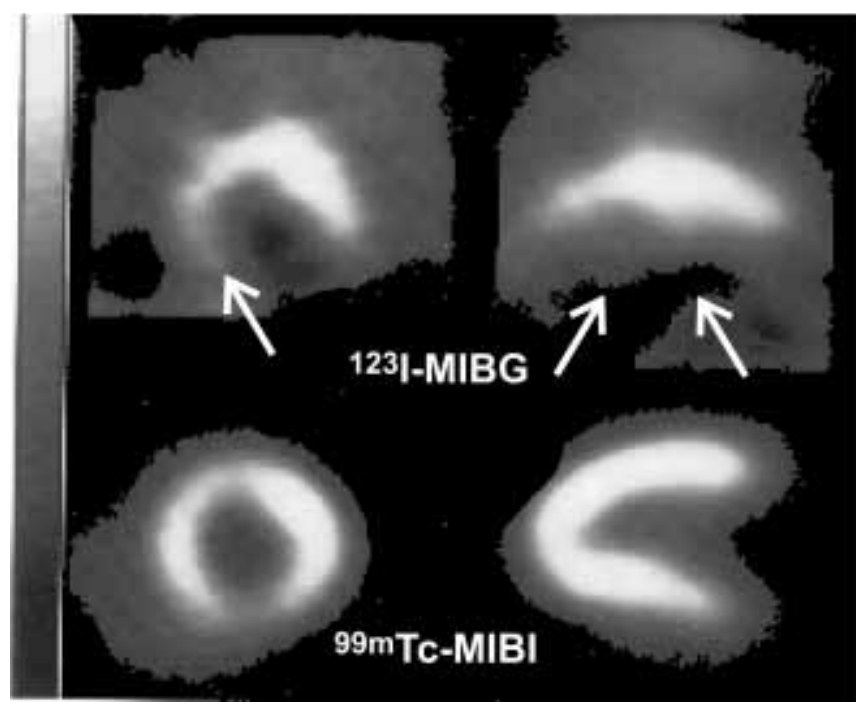

Fig. 10. Decreased uptake of ${ }^{123} \mathrm{I}-\mathrm{MIBG}$ in the posterior myocardium indicating a disorder of the autonomous cardiac innervation in a diabetic patient. The perfusion marker ${ }^{99 m}$ Tc-MIBI displays a normal perfusion. clear findings and in specifying the tumour type by utilising its specific ability of metabolic, receptor, and antigen imaging. Examples are iodine storing metastases of a thyroid malignancy and phosphate-sensitive bone metastases caused by many carcinoma. In many cases planar imaging is sufficient, whereas SPECT may be necessary to improve the spatial delineation. Unlike PET, which offers ${ }^{18}$ F-FDG to visualise a lot of different tumour types, SPECT must select that tracer which is appropriate for the tumour type to be investigated as indicated by the following examples. ${ }^{111} \mathrm{In}$-octreotide is appropriate for the visualisation of somatostatin receptor expressing tumours [54]. ${ }^{123} \mathrm{I}-\mathrm{MIBG}$ is also used for the diagnosis of pheochromocytoma and neuroblastoma [55]. ${ }^{67} \mathrm{Ga}$ has been applied in the follow-up of lymphoma treatment [56] and lung cancer [57]. Furthermore, the immunoscintigraphy of tumours is based on many different monoclonal antibodies labelled with ${ }^{99 m} \mathrm{Tc},{ }^{123} \mathrm{I}$, or ${ }^{111}$ In [58].

\section{Other SPECT applications}

SPECT has also been used to study liver diseases using ${ }^{99 m} \mathrm{Tc}$-sulphur-colloids [59] and ${ }^{99 m} \mathrm{Tc}-\mathrm{GSA}$ [60]. Cavernous hemangioma in the liver and other vascular abnormalities in the abdomen have been visualised with ${ }^{99 m} \mathrm{Tc}$-labelled blood cells [61, 62]. SPECT studies of the kidneys have been performed with ${ }^{99 m} \mathrm{Tc}$-DSMA as an indicator of the tubular renal function [63]. ${ }^{67} \mathrm{Ga}$ has been applied in different questions, e.g. in lung cancer [57], in lymphoma after treatment [56], and in different infections [64]. SPECT has provided important diagnostic and prognostic information for orthopaedic problems related to the lower extremities [65].

\section{PET applications}

Often PET is regarded just as a research tool which is too expensive to become of general importance for clinical routine. The change of this situation becomes obvious by the rapid world-wide increase of PET scanners from several dozens in 1990 to more than 300 in 2000. The short halflife of the mostly used positron emitters ranging from $2 \mathrm{~min}$ for ${ }^{15} \mathrm{O}, 10 \mathrm{~min}$ for ${ }^{13} \mathrm{~N}, 20 \mathrm{~min}$ for ${ }^{11} \mathrm{C}$ to $109 \mathrm{~min}$ for ${ }^{18} \mathrm{~F}$ and the necessity of a nearby cyclotron to produce these radionuclides is one of the reasons for the relatively slow expansion of PET. On the other hand ${ }^{18} \mathrm{~F}$-labelled deoxyglucose (FDG) has proved to be very valuable for many clinical applications and can be shipped from a cyclotron to PET sites within a radius of several hundred kilometres. Since 1979 FDG, which originated from Sokoloff's work with ${ }^{14} \mathrm{C}$-labelled deoxyglucose [66] for the study of cerebral glucose metabolism, has been successfully used in neurology, cardiology, and more recently especially in oncology.

\section{Oncological PET}

In many different tumours FDG-PET is able to visualise recurrent malignant tumours, their metastases and in some cases unknown primary tumours better than other imaging modalities such as CT or MRT [67]. Furthermore, FDGPET is useful in the follow-up of radio- or chemotherapy 


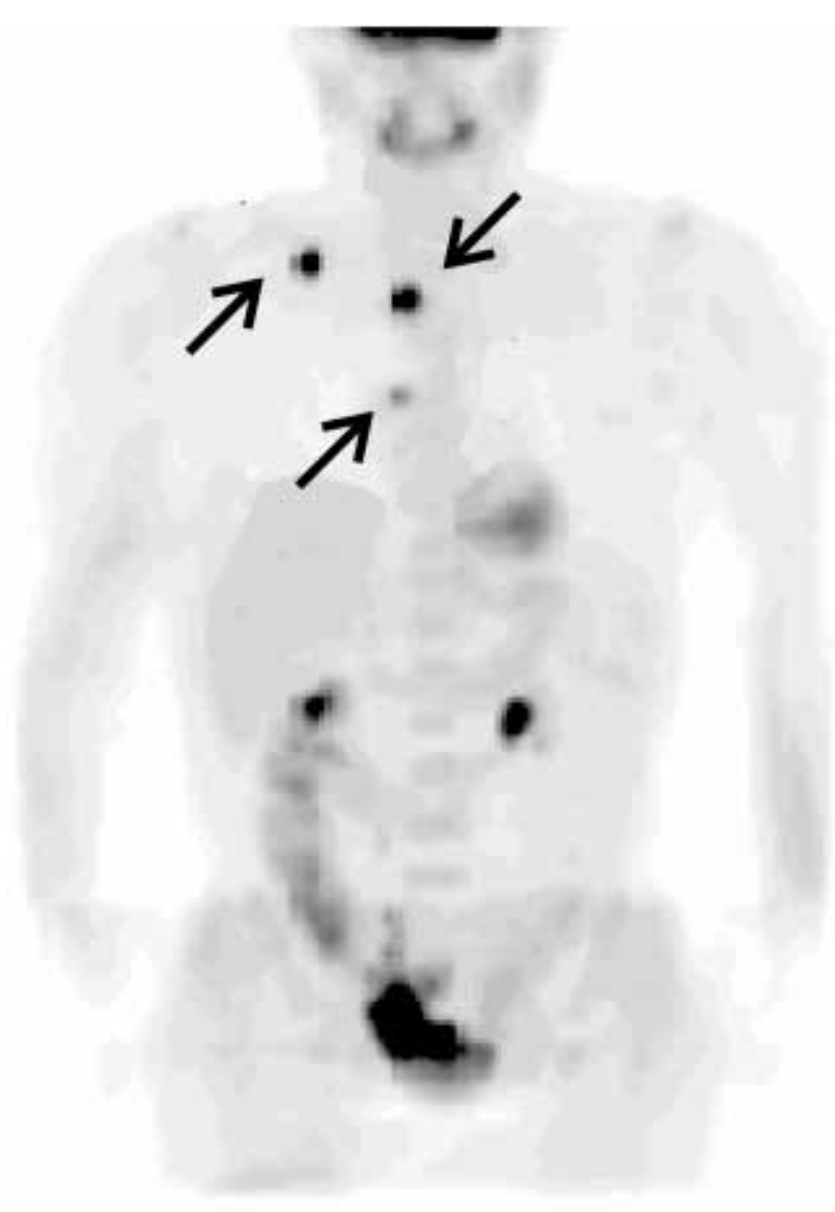

Fig. 11. The whole-body PET examination after the injection of FDG delineates three metastases (arrows) from prostate cancer not revealed by other imaging procedures.

when blood tumour markers indicate the existence of a recurrent or of metastases which cannot be delineated by CT or MRT. These advantages are the main reason for the recent world-wide increase of PET. Fig. 11 shows an example of the delineation of metastases not being seen by other methods.

\section{Neurological PET}

The primary use of FDG was in research and clinical studies of the brain $[68,69]$. Since the brain receives $90 \%$ of its energy by the oxidation of glucose, the glucose analogue FDG is able to indicate hypo- or hyperfunctions. Diminished uptake of FDG indicating cerebral hypofunction is found in epilepsy (Fig. 12), infarction, or dementia [70]. Because of the close coupling of cerebral blood flow and cerebral glucose consumption in normal tissue [71], but also in many diseases, the aim of FDG-PET is similar to that of perfusion studies with SPECT. Although many of these studies are done with SPECT nowadays, there is still a demand for PET if the specific question requires the still superior image resolution of PET. This may be the case when looking for a small epileptic hypometabolic focus [37].

If parts of the brain become very active compared to others because of an external or mental stimulation, these areas of hyperfunction can be delineated by increased up-

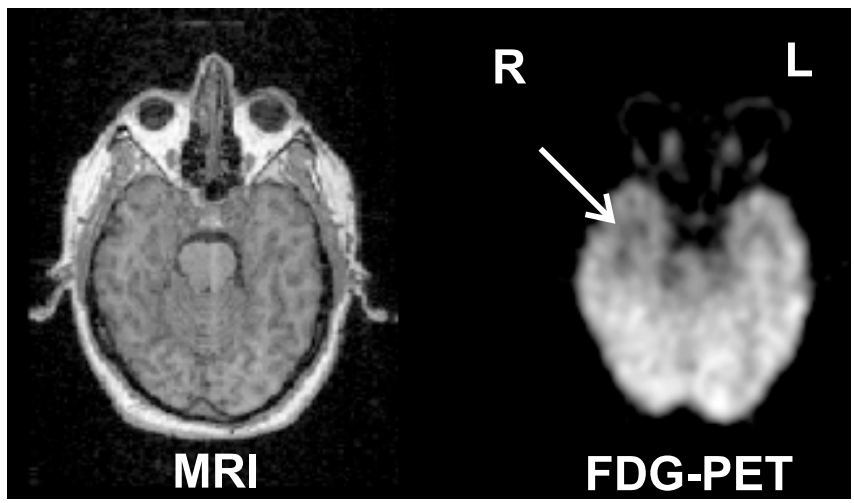

Fig. 12. Decrease of FDG in the area of the right temporal lobe delineating the suspected epileptic focus of a patient suffering from seizures. The image of magneto-resonance tomography (MCI) shows no disorder.

take of FDG. In the last years, such investigations have utilised ${ }^{15} \mathrm{O}$-water or ${ }^{15} \mathrm{O}$-butanol so that a sequence of different stimulations can be measured due to the rapid decay of the short-lived radionuclide ${ }^{15} \mathrm{O}$ (e.g. 1. rest, 2. slow thumb movements, 3. faster thumb movements, 4. very fast thumb movements). After intravenous injection these tracers are distributed within the brain in correlation to rCBF. Hundreds of such studies have been performed by neuroscientists so that many details of the motor and sensory systems as well as of higher cognitive processes could be delineated. An example of such a study which involves learning and memory [72] is given in Fig. 13. In addition to many questions related to basic research, neuroactivation studies can be advantageous for the single patient. For example, it is possible to delineate important language or motor control areas in the vicinity of a malignant brain tumour before it is removed by surgery.

There are hundreds of other positron-emitting radiopharmaceuticals, especially neurotransmitter ligands and amino acids which have been suggested for studies of the brain. Amino acids such as ${ }^{11} \mathrm{C}$-methionine (Fig. 9) are especially appropriate to help in the differential diagnosis of malignant brain tumours and in follow up studies [73]. Radiopharmaceuticals with an affinity to the neurotransmitter system have been applied in neurological and psychiatric research, e.g. in Parkinson's disease
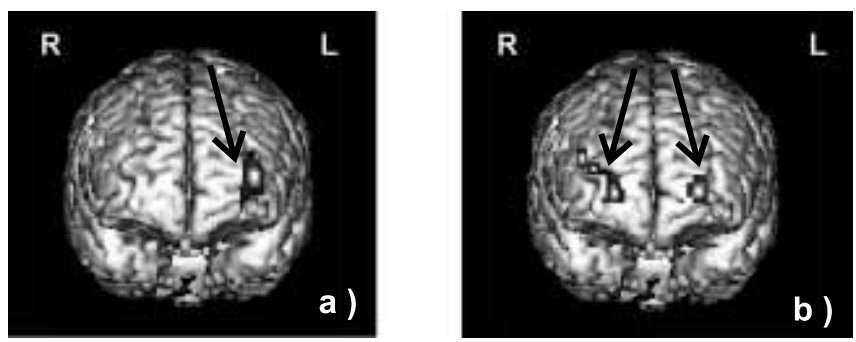

Fig. 13. During the learning (a) of uncorrelated word pairs (e.g. houseriver, piano-street) there is a maximum of increased blood flow in the left frontal cortex (arrow). During the retrieval (b) of one part of the word pairs there is an additional activation in the right frontal cortex (arrows). Only maxima of increased blood flow are displayed on a surface view of a standard MRI-image. 
and other motor disorders, in depression and schizophrenia [74-77]. Whereas first knowledge about the neurotransmitter system is usually acquired by animal research or post-mortem studies, PET can verify these findings in the human being in vivo. The radioactive labelling is often done with the positron emitter ${ }^{11} \mathrm{C}$ (half-life $=20 \mathrm{~min}$ ) so that the biochemical characteristics of the labelled transmitter molecules are unchanged [78]. The tracer kinetic analysis of the PET data leads to the determination of the binding potential, the density of neuroreceptors, and the binding affinity between receptor and drugs. Only few neurotransmitter ligands have reached clinical relevance such as ${ }^{18} \mathrm{~F}$-L-Dopa which is of help in the differential diagnosis of Parkinson's disease and in the follow up of the treatment [79]. A very interesting research area with possible implications to clinical routine is the study of the interaction between psychopharmaceuticals and specific PET compounds such as the dopamine receptor ligand ${ }^{11} \mathrm{C}$-raclopride [80] or the serotonergic receptor ligand ${ }^{18} \mathrm{~F}$ altanserin [81].

\section{Cardiological PET}

A third clinical field where PET has been successful is cardiology. Using blood flow indicators such as ${ }^{13} \mathrm{NH}_{3}$ [82], ${ }^{15} \mathrm{O}$-water [83], or ${ }^{82} \mathrm{Rb}$ [84] cardiac flow studies have been performed analogously to those done with SPECT (Fig. 14) [85]. Although PET yields a better resolution and is very favourable to combine a rest and an exercise study by using short-lived isotopes, just these isotopes have hindered the wide-spread use of this method due to the necessity of a nearby cyclotron or an expensive generator (for ${ }^{82} \mathrm{Rb}$ ). An even more important indication of cardiac PET is to define viable tissue in the neighbourhood of an infarction [86]. Since badly perfused myocardial tissue near to an infarction shows a relatively high uptake of FDG, this finding can be used to decide whether this tissue is still viable so that it will profit from a therapeutical intervention such as bypass surgery or PTCA. If the myocardial tissue is not viable, such an intervention is not necessary. As already seen in SPECT, fatty acids are used to study the myocardial energy metabolism. Here the natural amino acid palmitate is labelled with ${ }^{11} \mathrm{C}$ to study cardiomyopathy, for instance [87].

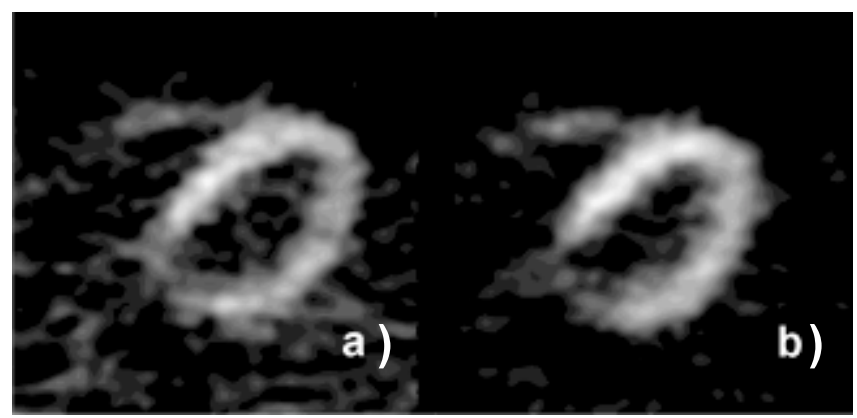

Fig. 14. PET images of myocardial uptake of the heart blood flow tracer ${ }^{13} \mathrm{~N}$-ammonia in a normal volunteer at rest (a) and during injection of dipyridamole (b) which increases blood flow similar to exercising. The increased blood flow is especially visualised by the lower background.

\section{SPECT vs. PET}

Although specific characteristics of SPECT and PET have already been compared above, some of the most important relationships and differences between these two tomographic modalities of nuclear medicine are to be summarised here. Because of the electronic collimation PET offers a better sensitivity with a factor up to 100 over SPECT. The possibility to do an accurate transmission measurement and an exact attenuation correction is the main reason for PET to be quantitative. In contrast SPECT is regarded as more qualitative yielding count rates rather than being calibrated with the physical entity of activity per volume. Nevertheless, there has been a considerable progress in the last years in improving the quantitative capabilities of SPECT. Because of the better sensitivity PET is able to record shorter time frames in dynamic studies. The radiolabelling of metabolically active molecules by the positron emitters ${ }^{11} \mathrm{C},{ }^{13} \mathrm{~N}$, or ${ }^{15} \mathrm{O}$ does not change the biochemical behaviour of the radiolabelled molecule so that the development of new radiopharmaceuticals can be based on prior biomedical knowledge. SPECT radiopharmaceuticals are considered being "unnatural" so that their introduction often needs more time, involving basic investigations and sometimes many vain trials. In spite of its quantitative and radiochemical advantages the availability of PET has remained rather limited due to the higher cost of the scanners and for the cyclotron and radiochemistry. As mentioned above, the progression of SPECT radiochemistry in the last years gave the possibility to transfer original experience made by PET research to the cheaper, more widely available and routinely used clinical tool SPECT. The only PET radiopharmaceutical for which SPECT does not offer an analogue is FDG, the working horse of PET. Here a bridge between PET and SPECT has been constructed by the introduction of dual-head coincidence gamma cameras. In addition to the normal SPECT mode, these double-headed SPECT systems offer the retraction of the mechanical collimators together with the combination of the electronics of the two gamma camera detectors by coincidence circuitry. In this way the SPECT instrument can also be used for PET imaging. Although the overall performance of such systems is inferior to dedicated PET systems they allow the use of FDG which has become especially important in oncology applications if the more cost-intensive PET instruments are not available.

Acknowledgment. The author thanks his colleagues Dr. M. Weckesser, Prof. K.-J. Langen, and Dr. B. J. Krause for providing images shown in Fig. 8, Fig. 9, Fig. 10, Fig. 12, and Fig. 13 and appreciates the secretarial help of Mrs. M. Bunn.

\section{References}

1. Kuhl, D. E., Edwards R. Q.: Image separation radioisotope scanning. Radiology 80, 653-662 (1963).

2. Rankowitz, S., Robertson, J. S., Higinbotham, W. A., Niell, A. M.: Positron scanner for locating brain tumors. IRE Int. Conv. Rec. 9, 49-56 (1962).

3. Cormack, A. M.: Representation of a function by its line integrals, with some radiological applications. II. J. Appl. Phys. 35, 29082913 (1964)

4. Hounsfield, G. N.: Computerized transverse axial scanning (tomography) Part I: Description of system. Brit. J. Radiol. 46, 1016-1022 (1973). 
5. Anger, H. O.: Multiple plane tomographic scanner. In: Tomographic imaging in Nuclear Medicine (Freedman, G. S. ed.). New York, Society of Nuclear Medicine, 1973, pp. 2-18.

6. Karp, J. S., Muehllehner, G., Mankoff, D. A., Ordonez, C. E., Ollinger, J. M., Daube-Witherspoon, M. E., Haigh, A. T., Beerbohm, D. J.: Continuous-slice PENN-PET: A positron tomograph with volume imaging capability. J. Nucl. Med. 31, 617-627 (1990).

7. Cherry, S.R., Dahlbom, M., Hoffman, E.J.: 3D PET using a conventional multislice tomograph without septa. J. Comput. Assist. Tomogr. 15, 655-668 (1991).

8. Budinger, T. F., Gullberg, G. T., Huesman, R. H.: Emission computed tomography. In: Image reconstruction from projections (Herman, G. T. ed.). Springer, Berlin 1979, pp. 147-246.

9. Radon, J.: Über die Bestimmung von Funktionen durch ihre Integralwerte längs gewisser Mannigfaltigkeiten. Sächsische Gesellschaft Wissenschaft, Leipzig, Math. Phys. 69, 262-277 (1917).

10. Ramachandran, G. N., Lakshminaraynan, A. V.: Three-dimensional reconstruction from radiographs and electron micrographs: Application of convolutions instead of Fourier transforms. Proc. Nat. Acad. Sci. 9, 2236 (1971).

11. Shepp, L. A., Vardi, Y.: Maximum likelihood reconstruction for emission tomography. Trans. Med. Imag. MI-1, 113-122 (1982).

12. Schmidlin, P., Bellemann, M. E., Brix, G.: Iterative reconstruction of PET images using a high-overrelaxation single-projection algorithm. Phys. Med. Biol. 42, 569-82 (1997).

13. Hudson, H. M., Larkin, R. S.: Accelerated image reconstruction using ordered subsets of projection data. IEEE Trans. Med. Imaging 20, 100-108 (1994).

14. Kay, J.: Statistical models for PET and SPECT data. Statistical Method in Med. Res. 3, 5-21 (1994).

15. Hoffman, E. J., Huang, S. C., Phelps, M. E.: Quantification in positron emission computed tomography: I. Effect of object size. J. Comput. Assist. Tomogr. 3, 299-308 (1979).

16. Defrise, M., Townsend, D., Clack, R.: Three-dimensional image reconstruction from complete projections. Phys. Med. Biol. 34, 573-587 (1989).

17. Kinahan, P. E., Rogers, J. G.: Analytic three-dimensional image reconstruction using all detected events. IEEE Trans. Nucl. Sci. 36, 964-968 (1989).

18. Daube-Witherspoon, M. E., Muehllehner, G.: Treatment of axial data in three-dimensional PET. J. Nucl. Med. 28, 1717-1724 (1987).

19. Defrise, M., Kinahan, P. E., Townsend, D. W., Michel, C., Sihomana, M., Newport, D. F.: Exact and approximate rebinning algorithms for 3-D PET data. IEEE Trans. Med. Imaging 16, 145158 (1997)

20. Bergström, M., Bohm, C., Ericson, K., Eriksson, L., Litton, J.: Corrections for attenuation, scattered radiation, and random coincidences in a ring detector positron emission transaxial tomograph. IEEE Trans. Nucl. Sci. 27, 435-444 (1980).

21. DeKemp, R. A., Nhamias, C.: Attenuation correction in PET using single photon transmission measurement. Med. Phys. 21, 771-778 (1994).

22. Karp, J. S., Muehllehner, G., Qu, H., Yan, X. H.: Singles transmission in volume-imaging PET with a ${ }^{137} \mathrm{Cs}$ source. Phys. Med. Biol. 40, 929-944 (1995).

23. Larsson, S. A.: Gamma camera emission tomography. Acta Radiol. (suppl. Stockholm) 363, 28-32 (1980).

24. Chang, L. T.: A method for attenuation correction in radionuclide computed tomography. IEEE Trans. Nucl. Sci. 25, 638-643 (1978).

25. Jaszczak, R. J., Greer, K. L., Floyd Jr., C. E., et al.: Improved SPECT quantification using compensation for scattered photons. J. Nucl. Med. 25, 893-900 (1984).

26. Msaki, P., Axelsson, B., Dahl, C. M., Larsson, S. A.: Generalized scatter correction method in SPECT using point scatter distribution functions. J. Nucl. Med. 28, 1861-1869 (1987).

27. Mukai, T., Links, J. M., Douglass, K. H., Wagner Jr., H. N.: Scatter correction in SPECT using non-uniform attenuation data. Phys. Med. Biol. 33, 1129-1140 (1988).

28. Ljungberg, M., Strand, S.-E.: Attenuation and scatter correction in SPECT for sources in a nonhomogeneous object: A Monte Carlo study. J. Nucl. Med. 32, 1278-1284 (1991).
29. Watson, C. C., Newport, D., Casey, M. E.: A single scatter simulation technique for scatter correction in 3D PET. In: Proc 1995 Int. Meeting Fully 3D-Image Reconstruction in Radiology and Nuclear Medicine (Grangeat, P., Amans, J. L. eds.). Kluwer Academic Publ, Dordrecht, 1996, pp. 215-219.

30. Stearns, C. W.: Scatter correction method for 3D PET using 2D fitted Gaussian functions. J. Nucl. Med. 36, 105P (1995).

31. Bergström, M., Eriksson, L., Bohm, C., Blomqvist, G., Litton, J.: Correction for scattered radiation in a ring detector positron camera by integral transformation of the projections. J. Comput. Assist. Tomogr. 7, 42-50 (1983).

32. Hoffman, E. J., Huang, S. C., Phelps, M. E., Kuhl, D. E.: Quantification in positron emission computed tomography: 4. Effect of accidental coincidences. J. Comput. Assist. Tomogr. 5, 391-400 (1981).

33. Harper, P. V., Beck, R., Charleston, D., Lathrop, K. A.: Optimization of a scanning method using ${ }^{99 m} \mathrm{Tc}$. Nucleonics 22, 50-54 (1964).

34. Novotnik, D. P., Canning, L. E., Cumming, S. A., Harrison, R. C., Higley, B., Nechvatal, G., Pickett, R. D., Piper, I. M., Bayne, V. J., Forster, A. M., Weisner, P. S., Neirinckx, R. D., Volckert, W. A., Troutner, D. E., Holmes, R. A.: Development of a ${ }^{99 m}$ Tc-labelled radiopharmaceutical for cerebral blood flow imaging. Nucl. Med. Commun. 6, 499-506 (1985).

35. Walovitch, R. C., Hill, T. C., Garrity, S. T., Cheeseman, E. H., Burgess, B. A., O'Leary, D. H., Watson, A. D., Ganey, M. V., Morgan, R. A., Williams, S. J.: Characterization of Technetium99m-L-ECD for brain perfusion imaging, Part I: Pharmacology of Technetium-99m-ECD in non human primates. J. Nucl. Med. 30, 1892-1901 (1989).

36. Winchell, H. S., Baldwin, R. M., Lin, T. H.: Development of I-123 labeled amines for brain studies: Localization of ${ }^{123} \mathrm{I}-$ iodophenylalcylamines in rat brain. J. Nucl. Med. 21, 940-946 (1980).

37. Duncan, J. S.: Imaging and epilepsy. Brain 120, 339-377 (1997).

38. O'Brien, T. J., So, E. L., Mullan, B. P., et al.: Subtraction ictal SPECT co-registered to MRI improves clinical usefulness of SPECT in localizing the surgical focus. Neurology 50, 445-454 (1998).

39. Maraganore, D. M., O'Connor, M. K., Bower, J. H., Kuntz, K. M., McDonnell, S. K., Schaid, D. J.; Rocca, W. A.: Detection of preclinical Parkinson disease in at-risk family members with use of $\left[{ }^{123}\right.$ I] beta-CIT and SPECT: an exploratory study. Mayo Clinic Proceedings 74, 681-685 (1999).

40. Kung, H. F., Alavi, A., Kung, M. P., Chang, W., Noto, R., Pan, S., Billings, J., Sorgentoni, K., Rauch, A., Reilley, J.: I-123-IBZM: A new CNS D2 receptor agent: Biodistribution and dosimetry in humans. J. Nucl. Med. 30, 834 (1989).

41. Buck, A., Westera, G., Sutter, M., Albani, C., Kung, H.F., von Schulthess, G.K.: Iodine-123-IBF SPECT evaluation of extrapyramidal diseases. J. Nucl. Med. 36, 1196-1200 (1995).

42. Höll, K., Deisenhammer, E., Dauth, J., Carmann, H., Schubiger, P. A.: Imaging benzodiazepine receptors in the human brain by Single Photon Emission Computed Tomography SPECT. Nucl. Med. Biol. 16, 757-763 (1989).

43. Kegeles, L. S., Mann, J. J.: In vivo imaging of neurotransmitter systems using radiolabeled receptor ligands. Neuropsychopharmacology 17, 293-307 (1997).

44. Langen, K.-J., Coenen, H. H., Roosen, N., Kling, P., Muzik, O., Herzog, H., Kuwert, T., Stöcklin, G., Feinendegen, L. E.: SPECT studies of brain tumors L-3-[ $\left.{ }^{123} \mathrm{I}\right]$ Iodo- $\alpha$-methyltyrosine ( ${ }^{123}$ IMT): First clinical results and comparison with PET and ${ }^{124}$ IMT. J. Nucl. Med. 31, 281-286 (1990).

45. Langen, K. J., Ziemons, K., Kiwit, J. C. W., Herzog, H., Kuwert, T., Bock, W. J., Stöcklin, G., Feinendegen. L. E., MüllerGärtner, H. W.: 3- $\left[{ }^{123}\right.$ I] Iodo-(-methyltyrosine and [methyl- $\left.{ }^{11} \mathrm{C}\right]-\mathrm{L}-$ methionine uptake in cerebral gliomas: A comparative study using SPECT and PET. J. Nucl. Med. 38, 517-522 (1997).

46. Wackers, F. J. Th., Berman, D. S., Maddahi, J., et al.: Technetium99m hexakis 2-methoxyisobutyl isonitrile: Human biodistribution, dosimetry, safety, and preliminary comparison to thallium-201 for myocardial perfusion imaging. J. Nucl. Med. 30, 301-311 (1989).

47. Jain, D., Wackers, F. J. Th., Mattera, J., et al.: Biokinetics of technetium-99m-tetrofosmin: myocardial perfusion imaging 
agent: Implications for a one-day imaging protocol. J. Nucl. Med. 34, 1254-1259 (1992).

48. Lebowitz, E., Greene, M. W., Fairchild, R., et al.: Thallium-201 for medical use, I. J. Nucl. Med. 16,151-155 (1975).

49. Höck, A., Freundlieb, C., Vyska, K., Lösse, B., Erbel, R., Feinendegen, L. E.: Myocardial imaging and metabolic studies with $17-{ }^{123}$ I-iodoheptadecanoic acid in patients with idiopathic congestive cardiomyopathy. J. Nucl. Med. 24, 22-28 (1983).

50. Knapp Jr., F. F., Kropp, J.: BMIPP-design and development. Int. J. Card. Imaging 15, 1-9 (1999).

51. Reske, S. N., Sauer, W., Machulla, H. J., Winkler, C.: 15-p-I-123iodophenyl-pentadecanoic acid as a tracer of lipid metabolism: Comparison with C-14-palmitate in murine tissues. J. Nucl. Med. 25, 1335-1342 (1984).

52. Corbett, J. R.: Fatty acids for myocardial imaging. Semin. Nucl. Med. 29, 237-258 (1999).

53. Sisson, J. C., Shapiro, B., Meyers, L. J., et al.: Meta-iodobenzylguanidine to map scintigraphically the adrenergic nervous system in man. J. Nucl. Med. 28, 1625-1636 (1987).

54. Lamberts, S. W. J., Bakker, W. H., Reubi, J.-C., Krenning, E. P.: Somatostatin-receptor imaging in the localization of endocrine tumours. N. Engl. J. Med. 323, 1246-1249 (1990).

55. Takahashi, N., Suzuki, T., Yamaya, K., Funyu, T.: The usefulness of $\left[{ }^{131} \mathrm{I}\right]$-metaiodobenzylguanidine $\left(\left[{ }^{131} \mathrm{I}\right]-\mathrm{MIBG}\right.$ scintigraphy performed one week after administration in diagnosing pheochromocytoma. Int. J. Urol. 6, 331-336 (1999).

56. Front, D., Israel, O.: The role of $67 \mathrm{Ga}$-scintigraphy in evaluating the results of therapy of lymphoma patients. Semin. Nucl. Med. 25, 60-71 (1995).

57. Chiti, A., Schreiner, F. A., Crippa, F., Pauwels, E. K., Bombardieri, E.: Nuclear medicine procedures in lung cancer. Eur. J. Nucl. Med. 26, 533-555 (1999).

58. Bischof Delaloye, A., Delaloye, B.: Tumor imaging with monoclonal antibodies. Semin. Nucl. Med. 25, 144-164 (1995).

59. Van Heertum, R. L., Yudd, A. P., Brunetti, J. C., Pennington, M. R., Gualtieri, N. M.: Hepatic SPECT imaging in the detection and clinical assessment of hepatocellular disease. Clin. Nucl. Med. 17, 948-953 (1992).

60. Roca, I., Ciofetta, G.: Hepatobiliary scintigraphy in current pediatric practice. Q. J. Nucl. Med. 42, 113-118 (1998).

61. Siegel, A., Mazurek, R.: Early dynamic SPECT acquisition for the imaging of hepatic hemangiomas utilizing Tc-99m labeled red blood cells. Clin. Nucl. Med. 22, 745-748 (1997).

62. Van Heertum, R. L., Brunetti, J. C., Yudd, A. P.: Abdominal SPECT imaging. Semin. Nucl. Med. 17, 230-246 (1987).

63. Williams, E. D.: Renal single photon emission computed tomography: Should we do it? Semin. Nucl. Med. 22, 112-121 (1992).

64. Palestro, C. J., Torres, M. A.: Radionuclide imaging of nonosseous infection. Q. J. Nucl. Med. 43, 46-60 (1999).

65. Etchebehere, E. C., Etchebehere, M., Gamba, R., Belangero, W., Camargo, E. E.: Orthopedic pathology of the lower extremities: Scintigraphic evaluation in the thigh, knee, and leg. Semin. Nucl. Med. 28, 41-61 (1998).

66. Sokoloff, L., Reivich, M., Kennedy, C., DesRosiers, M. H., Patlak, C. S., Pettigrew, K. D., Sakurada, O., Shinohara, M.: The $\left[{ }^{14} \mathrm{C}\right]$ deoxyglucose method for the measurement of focal cerebral glucose utilization: Theory, procedure and normal values in the conscious and anesthetized albino rat. J. Neurochem. 28, 897-916 (1977).

67. Strauss, L. G., Conti, P. S.: The applications of PET in clinical oncology. J. Nucl. Med. 32, 623-648 (1991).

68. Phelps, M. E., Huang, S. C., Hoffman, E. J., Selin, C., Sokoloff, L., Kuhl, D. E.: Tomographic measurement of local cerebral glucose metabolic rate in humans with (F-18)2-fluoro-2-deoxyD-glucose: Validation of method. Ann. Neurol. 6, 371-388 (1979).

69. Reivich, M., Kuhl, D., Wolf, A., Greenberg, J., Phelps, M., Ido, T., Casella, V., Fowler, J., Hoffman, E., Alavi, A., Som. P., Sokoloff, L.: The $\left[{ }^{18} \mathrm{~F}\right]$ fluorodeoxyglucose method for the measurement of local cerebral glucose utilization in man. Circ. Res. 44, 127-139 (1979).

70. Mazziotta, J.C., Phelps, M.E.: Positron emission tomography studies of the brain. In: Positron Emission Tomography and Autoradiography (Phelps, M. E., Mazziotta, J. C., Schelbert, H. R., eds.). Raven Press, New York 1986, p. 493.

71. Sokoloff, L.: Relationships among local functional activity, energy metabolism, and blood flow in the central nervous system. Fed. Proc. 40, 2311-2316 (1981).

72. Krause, B. J. Halsband, U., Schmidt, D., et al.: Neurofunctional correlates of encoding and retrieval in declarative associative learning. NeuroImage 5, S620 (1997).

73. Hübner, K. F., Purvis, J. T., Mahaley Jr., S. N., Robertson, J. T., Rogers, F., Gibbs, W. D., Partin, C. L.: Brain tumour imaging by positron emission computed tomography using ${ }^{11} \mathrm{C}$-labelled amino acids. J. Comput. Assist. Tomogr. 6, 544-550 (1982).

74. Burns, H. D., Hamill, T. G., Eng, W. S., Francis, B., Fioravanti, C., Gibson, R. E.: Positron emission tomography neuroreceptor imaging as a tool in drug discovery, research and development. Curr. Opin. Chem. Biol. 3, 388-394 (1999).

75. Farde, L.: The advantage of using positron emission tomography in drug research. Trends Neurosci. 19, 211-214 (1996).

76. Wong, W. F., Pearlson, G. D., Tune, L. E., Young, L. T., Meltzer, C. C., Dannals, R. F., Ravert, H. T., Reith, J., Kuhar, M. J., Gjedde, A.: Quantification of neuroreceptors in the living human brain: IV. Effect of aging and elevations of D2-like receptors in schizophrenia and bipolar illness. J. Cereb. Blood Flow Metab. 17, 331-342 (1997).

77. Kapur S. A.: New framework for investigating antipsychotic action in humans: Lessons from PET imaging. Mol. Psychiatry 3,135-140 (1998).

78. Sedvall, G., Farde, L., Nyback, H., Pauli, S., Persson, A., Savic, I., Wiesel, F. A.: Recent advances in psychiatric brain imaging. Acta Radiol. Suppl. 374, 113-115 (1990).

79. Garnett, E. S., Nahmias, C., Firnau, G.: Central dopaminergic pathways in hemiparkinsonism examined by positron emission tomography. Can. J. Neurol. Sci. 11, 174-179 (1984).

80. Farde, L., Hall, H., Ehrin, E., Sedvall, G.: Quantitative analysis of D2 dopamine receptor binding in the living human brain by PET. Science 231, 258-261 (1986).

81. Sadzot, B., Lemaire, C., Maquet, P., Salmon, E., Plenevaux, A., Degueldre, C., Hermanne, J. P., Guillaume, M., Cantineau, R., Comar, D., et al.: Serotonin 5HT2 receptor imaging in the human brain using positron emission tomography and a new radioligand, $\left[{ }^{18} \mathrm{~F}\right]$ altanserin: Results in young normal controls. J. Cereb. Blood Flow Metab. 15, 787-797 (1995).

82. Smith, G. T., Huang, S. C., Nienaber, C. A., Krivokapich, J., Schelbert, H. R.: Noninvasive quantification of regional myocardial blood flow with N-13 ammonia and dynamic PET. J. Nucl. Med. 29, 940 (1988).

83. Walsh, M. N., Bergmann, S. R., Steele, R. L., et al.: Delineation of impaired regional myocardial perfusion by positron emission tomography with $\mathrm{H}_{2}(15) \mathrm{O}$. Circulation 78, 612-620 (1988).

84. Gould, K. L., Goldstein, R. A., Mullani, N. A.: Economic analysis of clinical positron emission tomography of the heart with rubidium-82. J. Nucl. Med. 30, 707-717 (1989).

85. Herzog, H.: Basic ideas and principles for quantifying regional blood flow with nuclear medical techniques. Nuklearmedizin $\mathbf{3 5}$, 181-185 (1996)

86. Brunken, R., Schwaiger, M., Grover-McKay, M. et al.: Positron emission tomography detects tissue metabolic activity in myocardial segments with persistent thallium perfusion defects. J. Am. Coll. Cardiol. 10, 557-567 (1987).

87. Schelbert, H. R., Henze, E., Sochor, H., Grossman, R. G., Huang, S. C., Barrio, J. R., Schwaiger, M., Phelps, M. E.: Effects of substrate availability on myocardial C-11 palmitate kinetics by positron emission tomography in normal subjects and patients with ventricular dysfunction. Am. Heart J. 111, 1055-1064 (1986). 